\title{
Tuning of optimal P and PI controllers for level control of single tank system
}

\author{
S. Singh ${ }^{1 *}$, V. P. Singh ${ }^{1}$, S. P. Singh ${ }^{1}$, R. K. Dohare ${ }^{2}$ and S. Agarwal ${ }^{1}$ \\ Department of Electrical Engineering, National Institute of Technology, Raipur, India ${ }^{1}$ \\ Department of Chemical Engineering, National Institute of Technology, Jaipur, India ${ }^{2}$
}

\section{(C)2016 ACCENTS}

\begin{abstract}
In this paper, optimal P and PI controllers are proposed for level control of single tank system. The integral-square-error (ISE) of unit step response is considered as design criterion for obtaining optimal controller settings. This method of formulation of ISE requires only some alpha and beta parameters unlike integration with time. The teacher learner based optimization (TLBO) algorithm is employed for minimizing the performance index. It is found that the proposed TLBO based controllers provide excellent results in terms of time domain specifications.
\end{abstract}

\section{Keywords}

ISE, Level control, PID controller, Teacher learner based optimization, Tuning.

\section{Introduction}

Various methods have been proposed in the literature for obtaining optimal values of parameters of PID controller. Ziegler-Nichols (ZN) settings is one of the most used design criteria [1]. Other methods include integral of square time weighted error (ISTE), Pessen integral of absolute error (PIAE), Kessler Landau Voda (KLV), some overshoot rule (SO-OV), no overshoot rule (NO-OV), Mantz-Tacconi ZieglerNichols (MT-ZN) and refined Ziegler-Nichols (R$\mathrm{ZN})$ [2]. Other technique which provide better response in comparison to rule based tuning methods are PID controller tuning based on particle swarm optimization [4], Luus-Jaakola optimization procedure for PID controller tuning [3], PID controller tuning based on genetic algorithm [6], evolutionary computation based PID tuning [5] and PID tuning using soft computing techniques [7].In this work, optimal P and PI controllers are proposed for level control of single tank system using teacher learner based optimization technique. The integralsquare-error (ISE) is considered as performance index. The ISE is obtained in terms of alpha and beta parameters. Being simple and efficient; the teacher learner based optimization (TLBO) is used minimizing the performance index [8-11]. The TLBO is easy to implement due to absence of algorithmspecific parameters.

*Author for correspondence

165
The layout of this brief is as follows. Section 2 describes the system and its model. Section 3 provides the structure of controllers. The proposed method of tuning is discussed in section 4 . The algorithm utilized to minimize the ISE is detailed in section 5. Section 6 provides results. The paper is concluded in section 7 .

\section{The single tank system}

The block diagram shown in Figure 1 illustrates the liquid level control of single tank system [12].

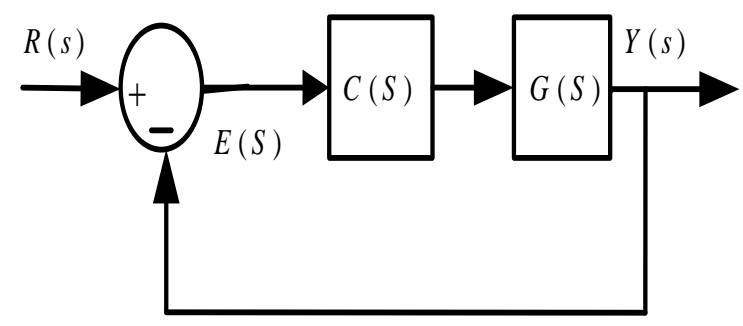

Figure 1 Single tank system

The transfer function of plant is given as

$G(s)=\frac{K}{\left(\tau_{a} s+1\right)}$

where, $K$ is gain and $\tau_{a}$ is time constant of tank. In block diagram, $C(s)$ represents the controller transfer function. 


\section{The controllers}

The transfer function of PID controller is

$$
C(s)=K_{p}\left(1+\frac{1}{T_{i} s}+T_{d} s\right)
$$

where, $K_{p}, T_{i}$ and $T_{d}$ are, respectively, the proportional gain, integral time constant and derivative time constant. The $\mathrm{P}$ and PI controller are given by (3)and (4), respectively.

$$
\begin{aligned}
& C(s)=K_{p} \\
& C(s)=K_{p}\left(1+\frac{1}{T_{i} s}\right)
\end{aligned}
$$

\section{The TLBO based tuning method}

In proposed work, the integral-square-error is considered as design criterion for tuning.

The ISE is given as

$$
J=\int_{t=0}^{t=\infty} e^{2}(t) d t
$$

This can be written as

$$
J=\frac{1}{2} \sum_{i=1}^{n} \frac{\beta_{i}^{2}}{\alpha_{i}}
$$

in terms of alpha and beta parameters [13] where $n$ is the order of error in $s$-domain. The alpha and beta parameters for error

$$
E(s)=\frac{b_{1} s^{n-1}+\cdots+b_{n}}{a_{0} s^{n}+a_{1} s^{n-1}+\cdots+a_{n}}
$$

\begin{tabular}{|c|c|c|c|c|}
\hline & $\begin{array}{l}d_{\mathrm{o}}^{1}=b_{1} \\
d_{\mathrm{o}}^{2}=b_{2}\end{array}$ & $\begin{array}{l}d_{2}^{1}=b_{3} \\
d_{2}^{2}=b_{4}\end{array}$ & $\begin{array}{l}d_{4}^{1}=b_{5} \\
d_{4}^{2}=b_{6}\end{array}$ & $\begin{array}{c}d_{6}^{1}=b_{7} \\
\ldots\end{array}$ \\
\hline$\beta_{1}=d_{\mathrm{o}}^{1} / c_{\mathrm{o}}^{1}$ & $d_{0}^{3}=d_{2}^{1}-\beta_{1} c_{2}^{1}$ & $d_{2}^{3}=d_{4}^{1}-\beta_{1} c_{4}^{1}$ & $d_{4}^{3}=d_{6}^{1}-\beta_{1} c_{6}^{1}$ & $\cdots$ \\
\hline$\beta_{2}=d_{0}^{2} / c_{0}^{2}$ & $d_{0}^{4}=d_{2}^{2}-\beta_{2} c_{2}^{2}$ & $d_{2}^{4}=d_{4}^{2}-\beta_{2} c_{4}^{2}$ & $d_{4}^{4}=d_{6}^{2}-\beta_{2} c_{6}^{2}$ & $\ddots$ \\
\hline$\beta_{3}=d_{0}^{3} / c_{0}^{3}$ & $d_{0}^{5}=d_{2}^{3}-\beta_{3} c_{2}^{3}$ & $d_{2}^{5}=d_{4}^{3}-\beta_{3} c_{4}^{3}$ & $\vdots$ & \\
\hline$\beta_{4}=d_{\mathrm{o}}^{4} / c_{\mathrm{o}}^{4}$ & $d_{\mathrm{o}}^{6}=d_{2}^{4}-\beta_{4} c_{2}^{4}$ & $\vdots$ & & \\
\hline $\begin{array}{c}\beta_{5}=d_{0}^{5} / c_{0}^{5} \\
\vdots\end{array}$ & $\vdots$ & & & \\
\hline
\end{tabular}

are given in Table 1 and Table 2 .

Table 1 Alpha table

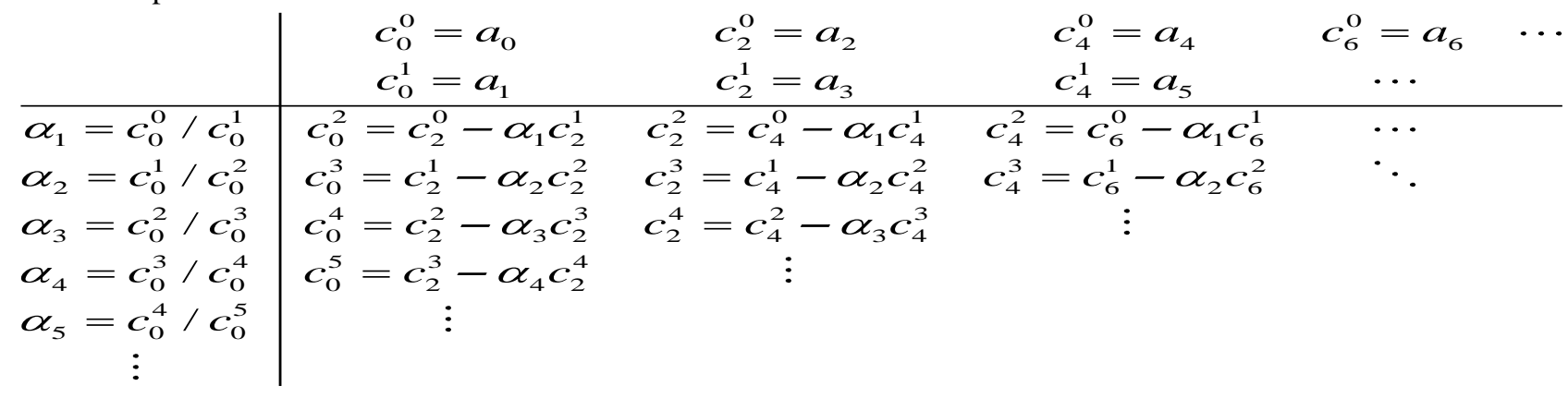

Table 2 Beta table

The error obtained in $s$-domain for system given in Figure 1 can be written as

$$
E(s)=R(s)-Y(s)=\frac{R(s)}{1+G(s) C(s)}
$$

where,

$$
R(s)=\frac{1}{s}
$$

\section{Teacher learner based optimization (TLBO) algorithm}

Recently, Rao et al. proposed TLBO algorithm [14]. This algorithm is composed of two phases namely teacher phase and learner phase. In former phase, the class teacher tries to improve the average performance of the class. In latter phase, learners compete among themselves and try to enhance their performance. 
Suppose, the number of learners and subjects offered in the class are a total of $R$ and $C$, respectively. The performance of $i$ th learner, $i=1,2, \cdots, R$, in $j$ th subject, $j=1,2, \cdots, C$ is given as $X_{i, j}$. In teacher phase, class performance is updated by:

new $X_{i, j}=X_{i, j}+r\left(X_{\text {teacher }, j}-T_{f} \bar{X}_{i, j}\right)$

Where $n e w X_{i, j}$ is updated performance of the class and $r$ is a random number in the range $(0,1)$. $X_{\text {teacher }, j}$ and $\bar{X}_{i, j}$ denote respectively the performance of class teacher (i.e. the best learner of the class) and mean performance of the class. The parameter $T_{f}$ is called teacher factor which is given by

$T_{f}=\{1,2\}$

At the end of teacher phase, new $X_{i, j}$ is updated if it denotes a better. In learner phase, learners' performance is updated

$n e w X_{i, j}=X_{i, j}+r\left(X_{u, j}-X_{v, j}\right)$

Where $u$ and $v$ are randomly selected learners such that $u \neq v, f\left(X_{u, j}\right)<f\left(X_{v, j}\right)$ and $r$ is a random number such that $r=(0,1)$. At the end of learner phase, the solution $n e w X_{i, j}$ is updated in $X$ if it represents better solution. This completes teacher and learner phases of TLBO algorithm. These two phases are repeated until the termination criterion achieves.
The parameters [12] of single tank system are $K=5$ and $\tau_{a}=2$.

P controller:

For the system given by(1), the error transfer function (7) becomes

$$
E(s)=\frac{b_{1} s+b_{2}}{a_{0} s^{2}+a_{1} s+a_{2}}
$$

where,

$$
\begin{aligned}
& a_{0}=\tau_{a} \\
& a_{1}=K K_{p}+1 \\
& a_{2}=0 \\
& b_{1}=\tau_{a} \\
& b_{2}=1
\end{aligned}
$$

The alpha and beta tables (Table 1 and Table 2) modifies to Table 3 and Table 4, respectively.

Table 3 Alpha table

$$
\begin{array}{l|ll} 
& c_{0}^{0}=a_{0} & c_{2}^{0}=a_{2} \\
& c_{0}^{1}=a_{1} & c_{2}^{1}=0 \\
\hline \alpha_{1}=c_{0}^{0} / c_{0}^{1} & c_{0}^{2}=c_{2}^{0} & \\
\alpha_{2}=c_{0}^{1} / c_{0}^{2} & &
\end{array}
$$

Table 4 Beta table

$$
\begin{array}{l|ll} 
& d_{0}^{1}=b_{1} & d_{2}^{1}=0 \\
& d_{0}^{2}=b_{2} & d_{2}^{2}=0 \\
\hline \beta_{1}=d_{0}^{1} / c_{0}^{1} & d_{0}^{3}=0 \\
\beta_{2}=d_{0}^{2} / c_{0}^{2} &
\end{array}
$$

\section{Results and discussion}

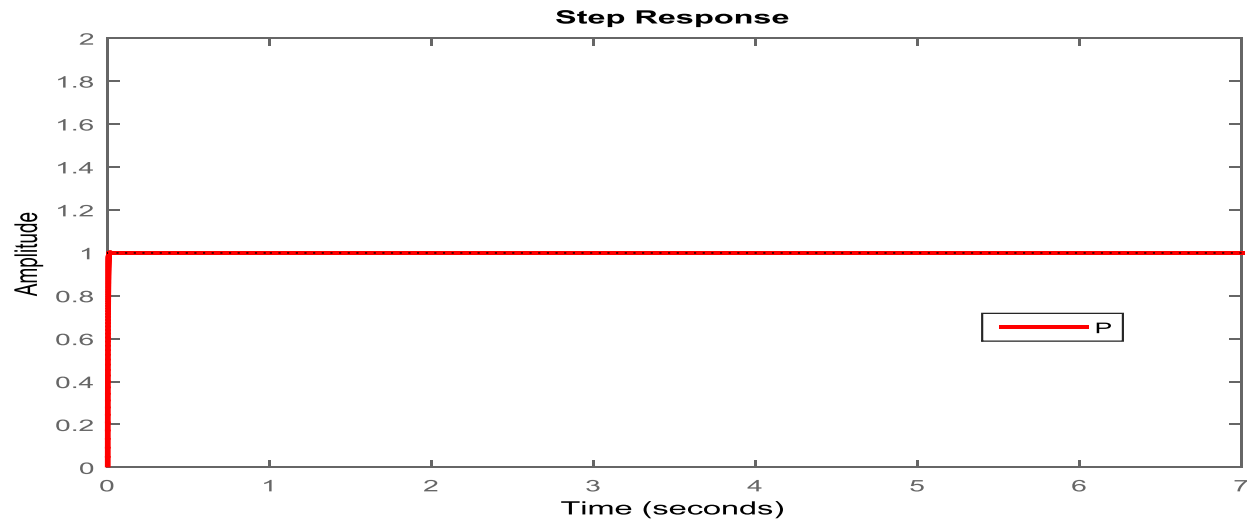

Figure 2 Step response of the system

The performance index, given by(6), becomes

$J=\frac{1}{2} \sum_{i=1}^{2} \frac{\beta_{i}^{2}}{\alpha_{i}}=\frac{1}{2}\left\{\frac{\beta_{1}^{2}}{\alpha_{1}}+\frac{\beta_{2}^{2}}{\alpha_{2}}\right\}$ 
Minimizing (20) using TLBO, the optimal value of controller gain obtained is $K_{p}=257.96$. Figure 2 provides the unit step response for the proposed setting. The settling time and peak over shoot are 0.006 seconds and $0 \%$ respectively.

PI controller:

For (1), the error transfer function (7) turn out to be

$$
E(s)=\frac{b_{1} s+b_{2}}{a_{0} s^{2}+a_{1} s+a_{2}}
$$

where,

$$
\begin{aligned}
& a_{0}=T_{i} \tau_{a} \\
& a_{1}=T_{i}\left(K K_{p}+1\right) \\
& a_{2}=K K_{p} \\
& b_{1}=T_{i} \tau_{a} \\
& b_{2}=T_{i}
\end{aligned}
$$

The alpha and beta tables (Table 1 and Table 2) become, respectively, Table 5 and Table 6.

Table 5 Alpha table

$$
\begin{array}{l|ll} 
& c_{0}^{0}=a_{0} & c_{2}^{0}=a_{2} \\
& c_{0}^{1}=a_{1} & c_{2}^{1}=0 \\
\hline \alpha_{1}=c_{0}^{0} / c_{0}^{1} & c_{0}^{2}=c_{2}^{0} & \\
\alpha_{2}=c_{0}^{1} / c_{0}^{2} & &
\end{array}
$$

Table 6 Beta table

$$
\begin{array}{l|ll} 
& d_{0}^{1}=b_{1} & d_{2}^{1}=0 \\
& d_{0}^{2}=b_{2} & d_{2}^{2}=0 \\
\hline \beta_{1}=d_{0}^{1} / c_{0}^{1} & d_{0}^{3}=0 \\
\beta_{2}=d_{0}^{2} / c_{0}^{2} &
\end{array}
$$

The performance index, given by(6), modifies to

$$
\begin{aligned}
& J=\frac{1}{2} \sum_{i=1}^{2} \frac{\beta_{i}^{2}}{\alpha_{i}}=\frac{1}{2}\left\{\frac{\beta_{1}^{2}}{\alpha_{1}}+\frac{\beta_{2}^{2}}{\alpha_{2}}\right\} \\
& J=\frac{1}{2}\left\{\frac{b_{1}^{2}}{a_{0} a_{1}}+\frac{b_{2}^{2}}{a_{1} a_{2}}\right\}
\end{aligned}
$$

Minimizing (28) using TLBO, the optimal values of controller gains obtained are $K_{p}=100$ and $T_{i}=8.03 e-12$. Figure 3 shows the step response obtained due to proposed settings. The settling time and peak over shoot obtained are 0.003 seconds and $99.40 \%$, respectively.

From the proposed P and PI controllers, it is clear that the TLBO based tuning provides excellent results in terms of time domain specifications.

\section{Conclusion}

In this work, optimal $\mathrm{P}$ and PI controllers are proposed for level control of single tank system. The controller settings are obtained by minimizing the ISE of unit step response. This formulation of ISE requires a fixed number of alpha and beta parameters only. The teacher learner based optimization algorithm is employed for minimizing the performance index. The obtained controller settings provided excellent time response.

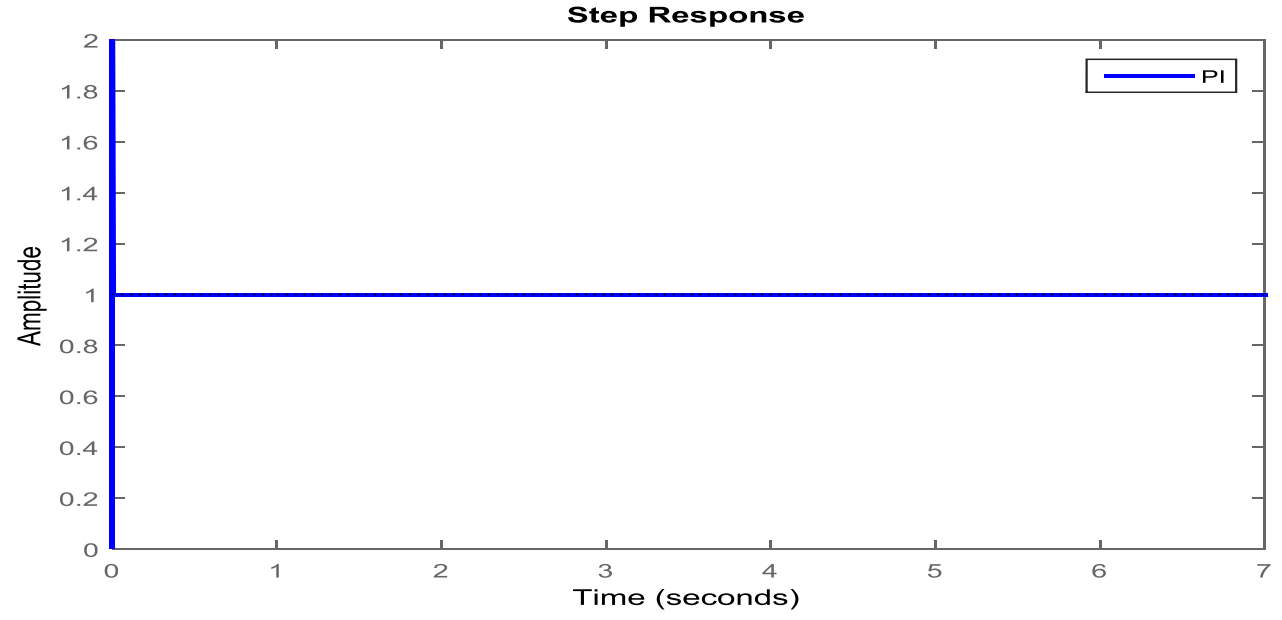

Figure 3 Step response of the system 


\section{Acknowledgment}

None.

\section{Conflicts of interest}

The authors have no conflicts of interest to declare.

\section{References}

[1] Ziegler JG, Nichols NB. Optimum settings for automatic controllers. trans. ASME.1942; 64(11).

[2] McCormack AS, Godfrey KR. Rule-based autotuning based on frequency domain identification. IEEE Transactions on Control Systems Technology. 1998; 6(1):43-61.

[3] Rathore NS, Chauhan DP, Singh VP. Luus-Jaakola optimization procedure for PID controller tuning in reverse osmosis system. In proceedings 23rd IRF international conference, Chennai, India 2015 (pp. 125).

[4] Rathore NS, Kundariya N, Narain A. PID controller tuning in reverse osmosis system based on particle swarm optimization. International Journal of Scientific and Research Publications. 2013; 3(6):1-5.

[5] Wadhwani S, Verma V. Evolutionary computation techniques based optimal PID controller tuning. International Journal of Engineering Trends and Technology. 2013; 4(6): 2529-34.

[6] Bindu R, Namboothiripad MK. Tuning of PID controller for DC servo motor using genetic algorithm. International Journal of Emerging Technology and Advanced Engineering. 2012; 2(3):310-4.

[7] Nagaraj B, Subha S, Rampriya B. Tuning algorithms for PID controller using soft computing techniques. International Journal of Computer Science and Network Security. 2008; 8(4):278-81.
[8] Baykasoğlu A, Hamzadayi A, Köse SY. Testing the performance of teaching-learning based optimization (TLBO) algorithm on combinatorial problems: Flow shop and job shop scheduling cases. Information Sciences. 2014; 276:204-18.

[9] Singh M, Panigrahi BK, Abhyankar AR. Optimal coordination of directional over-current relays using teaching learning-based optimization (TLBO) algorithm. International Journal of Electrical Power \& Energy Systems. 2013; 50:33-41.

[10] Rao RV, Kalyankar VD. Parameters optimization of advanced machining processes using TLBO algorithm. EPPM, Singapore. 2011; 20:21-31.

[11] Zhile YA, Kang LI, Qun NI, Yusheng XU, Foley A. A self-learning TLBO based dynamic economic/environmental dispatch considering multiple plug-in electric vehicle loads. Journal of Modern Power Systems and Clean Energy. 2014; 2(4):298-307.

[12] Bhanot S. Process control: principles and application. Oxford University Press; 2008.

[13] Hutton M, Friedland B. Routh approximations for reducing order of linear, time-invariant systems. IEEE Transactions on Automatic Control. 1975; 20(3):32937.

[14] Rao RV, Savsani VJ, Vakharia DP. Teachinglearning-based optimization: a novel method for constrained mechanical design optimization problems. Computer-Aided Design. 2011; 43(3):303-15. 